\title{
Using zebrafish as the model organism to understand organ regeneration
}

\author{
SHI WenChao, FANG ZhiBing, LI Li \& LUO LingFei* \\ Key Laboratory of Freshwater Fish Reproduction and Development, Ministry of Education, Laboratory of Molecular Developmental Biology, \\ School of Life Sciences, Southwest University, Chongqing 400715, China
}

Received October 27, 2014; accepted January 27, 2015

\begin{abstract}
The limited regenerative capacity of several organs, such as central nervous system (CNS), heart and limb in mammals makes related major diseases quite difficult to recover. Therefore, dissection of the cellular and molecular mechanisms underlying organ regeneration is of great scientific and clinical interests. Tremendous progression has already been made after extensive investigations using several model organisms for decades. Unfortunately, distance to the final achievement of the goal still remains. Recently, zebrafish became a popular model organism for the deep understanding of regeneration based on its powerful regenerative capacity, in particular the organs that are limitedly regenerated in mammals. Additionally, zebrafish are endowed with other advantages good for the study of organ regeneration. This review summarizes the recent progress in the study of zebrafish organ regeneration, in particular regeneration of fin, heart, CNS, and liver as the representatives. We also discuss reasons of the reduced regenerative capacity in higher vertebrate, the roles of inflammation during regeneration, and the difference between organogenesis and regeneration.
\end{abstract}

organ regeneration, zebrafish, fin, heart, liver, CNS

Citation: Shi WC, Fang ZB, Li L, Luo LF. Using zebrafish as the model organism to understand organ regeneration. Sci China Life Sci, 2015, 58: 343-351, doi: $10.1007 / \mathrm{s} 11427-015-4838-\mathrm{z}$

Regeneration is the restoration of organ mass, structure, and function after damage, which is indispensable for human health. Unfortunately, the regenerative capacity obviously attenuates along with the evolution. The salamanders, newts, hydra, flatworms and lower vertebrates such as urodele amplibians and teleosts possess powerful regenerative capacity for nearly all the organs [1]. However, the mammals including human beings obtain variable regenerative potential in different organs [2]. These organs are mainly sub-divided to three types according to their regenerative potential: those with high and constant regenerative capacity, such as skin and blood; those can regenerate well after injury, such as the liver, skeletal muscle and bone; and those with low regenerative potential, including the neural tissues, heart

*Corresponding author (email: 1luo@swu.edu.cn) and limb [3]. In cases of neuron degeneration, heart failure, limb disability, etc., the limited regenerative capacity of these organs can cause severe outcomes, which lead to the heavy social-economic burden. Thus, development of approaches to promote organ regeneration has attracted considerable scientific and clinical efforts.

Tremendous efforts have been paid and two strategies have been developed to restore the organ mass. One is the stem cell-based approach which sheds light on the production of therapeutic cells. Although some clinical trials have been carried out, the therapeutic effects and underlying mechanisms are still under intensive evaluation. Another approach is focusing on the proper regeneration animal models with the goal of understanding cellular and molecular mechanisms in details so that to develop translatable therapies [2]. This approach may help to induce the endo- 
genous regenerative capability via circumventing the limitations in mammals. To achieve this goal, Zebrafish (Danio rario) animal model is recently popularly used to study regeneration, taking advantages of its better regenerative capacity than mice and humans. Zebrafish is a sweet water pet fish which were brought into the lab by Streisinger, a scientist in genetics [4]. It eventually became an attractive animal model after contributions of three large genetic screen by Nüsslein-Volhard, Driever, and Fishman [5-7]. This tidy pet has several unique advantages, for example, good reproduction ability and large number of progenies suitable for large scale screen, rapid extra-uterine development for easy observation and manipulations, and optic transparence proper for the continual in vivo time-lapse imaging. $[1,3]$. Many of its organs, in particular those that can hardly regenerate in mammals, such as the CNS, heart, and limb $[2,3,8-14]$, are able to regenerate. Therefore, it is a model organism ideal for the study of the cellular and molecular events of organogenesis and regeneration.

\section{Organ injury approaches applied in zebrafish}

Several methods have been applied in zebrafish to damage or ablate the organs, followed by regeneration. These applications range from surgical removal of partial organs to killing most of the targeted cell types. Each method has its advantages and disadvantages. Physical surgery is commonly applied to the organs that are easily accessed, such as the fin, liver and brain $[3,11,13]$. These manipulations are quite simple and feasible, but meanwhile, sacrifice the precision and repeatability. Different severity and injured position of the lesion usually result in distinct outcomes. For example, in liver regeneration, larger damage of the adult liver lobes usually makes fish die quickly. Other physical injuries are also applied, for example, laser targeted cell ablation is mainly used to induce lesions of retina photoreceptor and heart muscles recently $[8,15]$. Chemical injury is based on the toxicity of the compounds to the target organs. This method is mainly used in the liver and brain because several types of chemicals are toxic to them [13]. However, these chemicals also show side effects even the proper dosage is used, which somewhat prevent the investigations. Recently, the transgenic approaches in combination with chemicals become widely used. The basic principle of these approaches is the generation of transgenic line driving tissue-specific protein expression, which allows targeted cell ablation under certain conditions. Several types of these proteins have been developed, for example, Diphteria toxin A, bacterial toxin Kid, and Killer Red [16-18]. To achieve that the toxic factors can be conditionally induced, a new approach has recently been developed. This method takes advantage of an bacterial enzyme nitroreductase (NTR) that catalyzes a pro-drug metronidazole (Mtz) into a cytotoxic product, thus crosslinking DNA and inducing cell death
[19]. Therefore, by placing the NTR cassette under the control of a tissue-specific promoter and generating a stable transgenic line [20,21], targeted cell ablation can be achieved by the addition of Mtz at any desired time point $[20,21]$. However, the variable efficiencies of NTR in different cell types require careful titrations in different applications. Although each approach has its own disadvantages, combinations of them may provide considerable contributions in the regeneration study using zebrafish as the model system.

\section{Organ regeneration in zebrafish}

Many organs in zebrafish are able to regenerate. Studies have covered nearly all the important organs, such as the fin, heart, CNS, liver, pancreas, kidney, bone, and hair cells $[3,12,22]$. These studies have provided tremendous progress in the understanding of cellular and molecular mechanisms underlying organ regeneration. In this review, we will focus on recent progress in the regeneration of the fin, heart, CNS and liver in zebrafish, which have been extensively investigated.

\subsection{Fin regeneration}

Fin, in particular the adult caudal fin regeneration, is primarily and intensively studied in zebrafish because of its simple structure, easy external surgeries and rapid regeneration [2,11]. By means of caudal fin amputation, previous studies indicated that the injured fin could quickly regenerate to its pre-amputation situation within approximately 2-3 weeks in adults and only 3 days in the larvae [2,11]. The whole regeneration process is currently divided into four phases (Figure 1). The first phase is the wound healing which initiates immediately after injury. The important structure, apical epidermal cap (AEC) is quickly formed at this period through migration of the non-proliferative lateral epithelial cells over the wound [23] (Figure 1). The AEC is crucial for the subsequent formation of blastema, the essential organization to start and promote fin regeneration [24]. The second phase (Figure 1), formation of blastema, is accomplished by the dedifferentiation and proliferation of cells in the disorganized mesenchymal tissue under AEC [23]. The signals from wound epidermis are important for this process. The appearance of blastema is the hallmark of fin regeneration because this structure is not formed under physiological condition. It is an accumulated mass of pluripotent progenitor cells that could give rise to a variety of cell types in the regenerating tissues. The blastema can be further sub-divided into the proximal and distal blastema based on the proliferation rate and the expression of $m s x b$, a key marker of blastema [23]. After establishment of the blastema, the third phase, regenerative outgrowth initiates, which is characterized by the extensive proliferation of cells 


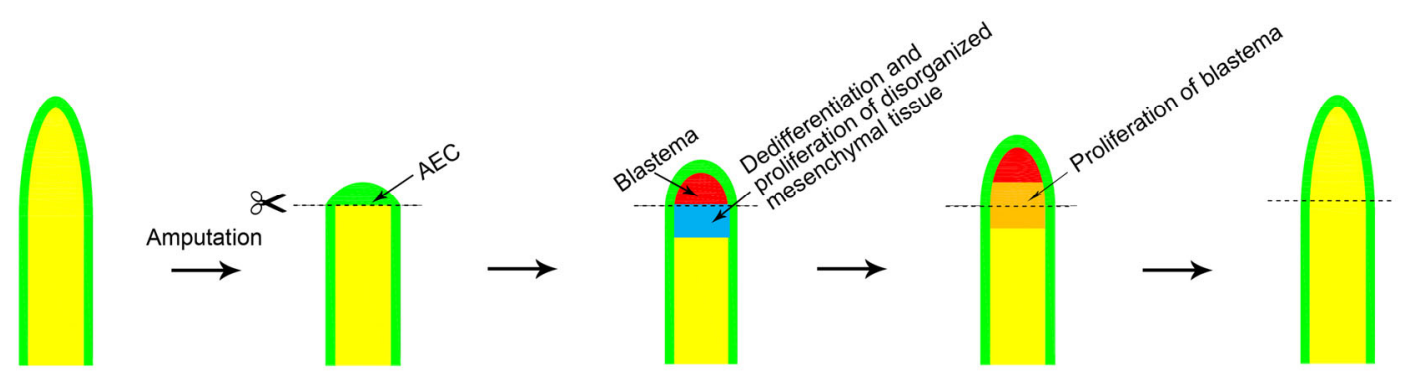

Figure 1 (color online) Schematic cartoon shows the regeneration process of the amputated fin. Once the fin is amputated, apical epidermal cap (AEC) is quickly formed to cover the wound. Subsequently, the blastema is accomplished by the dedifferentiation and proliferation of cells in the disorganized mesenchymal tissue under AEC. The proliferation of blastema ensures the growth of the newly generated fin. Eventually, the amputated fin regenerated to its original length.

in the proximal blastema (Figure 1). The newly proliferated cells quickly move to the appropriate locations to restore the tissues with mesenchymal cells and fibroblasts. When the newly generated fin grows to its original length, the final phase, regenerative termination ensues (Figure 1). But the signals and mechanisms responsible for this termination stage are still unknown [25]. Recently, the study on the adult fin regeneration after amputation indicated that the cellular source of the regenerated fins was the resident osteoblasts [26]. Different to that of embryonic stages, the adult fins are actually the appendage of the bone. Meanwhile, other bone structure, including the skull, could also be regenerated from the dedifferentiation of osteoblasts [27]. Thus, these studies shed light on the deep knowledge of the restore of bone after injury, which will be quite helpful to the therapies of bone injury in humans.

Consistent with the cellular events in the whole process, the molecular mechanisms have been largely unraveled. The most important signal network that has been intensively investigated is the Wnt signaling pathway [28], a well-known signal pathway crucial for multiple biological processes during early development, organogenesis, tumorigenesis and so on. The importance of Wnt in zebrafish fin regeneration was primarily revealed by the functional study of its target lef, which was firstly and impressively expressed in the wound epithelium as early as 12 hours post amputation (hpa) [29]. The application of Wnt signaling inhibitor Dkk1 or overexpression of Wnt5b through heat shock inducible line blocked the fin regeneration [2,28,30]. Further studies indicated that Wnt5b signaling was required for the specification of the blastemal mesenchyme and basal epithelial layer. Heat shock induced expression of Dkk resulted in defects in blastema and the distorted expression of lefl and msxb [28,30]. Consequently, the regenerative outgrowth was blocked. This conclusion was further supported by the application of several chemicals that could inhibit Wnt signaling [31,32]. Fgf signaling is also involved in the fin regeneration, which is believed to function downstream of Wnt. Knockdown of Fgf signaling using a dominant negative $f g f r l$ transgenic line impaired fin regeneration [33], and mutant of the Fgf family member $f g f 20 a$ failed to initi- ate regeneration [34]. Retinoic acid (RA) and hedgehog (Hh) signaling are also suggested to play important roles in the fin regeneration, in particular in the patterning of the regenerating issues $[2,35,36]$. Recently, the orchestrated roles of Wnt, Fgf, RA and Hh signaling networks in the fin regeneration were elucidated by Daniel Wehner and colleagues [37], whose work indicated that Wnt signaling in the nonproliferative distal blastema was required for cell proliferation in the proximal blastema and this signaling acted through Fgf and Bmp signaling to control epidermal patterning, whereas RA and Hh signals mediated its effects on blastemal cell proliferation [37]. Meanwhile, the function of noncoding RNAs in the fin regeneration attracts attention [38]. Through the knockdown assays, several groups presented the data that miR-203 and miR-133 took part in the regulation of fin regeneration via controlling the Wnt and Fgf signaling pathways [39,40]. Additionally, low function of Dicer, a key component of the miRNA synthesis machinery, showed negative effects on the fin regeneration [38]. Additional study provided evidences that upregulation of autophagy in the regeneration zone was required for the zebrafish caudal fin regeneration through preventing cells from undergoing apoptosis [41]. Calcineurin-mediated mechanism was important in the regulation of coordinated outgrowth of zebrafish regenerating fins [42]. All these studies expanded our knowledge on the cellular and molecular events of fin regeneration.

\subsection{Heart regeneration}

Different from mammals, adult zebrafish heart can regenerate after damage, which has attracted increasing attention [10]. The initial studies focused on the ventricle of adult heart. Adult zebrafish ventricle is composed of three different muscle lineages, primordial, trabecular and cortical muscle $[14,43]$. Usually, the heart ventricle is partially injured by surgery (Figure 2A). Taking advantage of the surgery, lineage tracing and transplantations, investigators revealed origins of the regenerating heart tissues. It is believed that the majority of the regenerating myocardium comes from the resident $\mathrm{cmcl}^{+}$cardiomyocytes [44,45] 
(Figure 2A), but not epicardium or epicardial-derived cells $[46,47]$. Further study using the rainbow assay [43] indicated that during the first 30 days post injury, cortical muscle was the major components reconstructing the muscular wall. However, contribution of primordial muscle could still be detectable at this stage. Thus, after 60 days when the tissue was restored, both primordial and cortical muscle layers played roles and the cortical muscle was the major player $[14,43]$. The order of both muscle layers in this regeneration process is opposed to their sequences in heart development $[14,43]$. Similar to that in the fin regeneration, the dedifferentiation of the cardiomyocyte of the injury sites is the dominant mechanism of zebrafish heart regeneration, because the cardiomyocytes present structural alterations after injury and the regenerating myocytes begin to express gata4, a gene essential for cardiac development [45]. Subsequently, these dedifferentiated cardiomyocyte functions as the cell source to support the regenerating tissue through extensive proliferation (Figure 2A). During the process, epicardial cells also show functions to facilitate generation of new vascular components $[1,14,46]$. In addition to the dedifferentiation mechanism when adult heart is partially damaged, recent study applied the transgenic-based assay to ablate large parts of the larvae heart (Figure 2B). Transdifferentiation was shown to be the key mechanism under this condition [48]. This model took advantage of a transgenic line in which nitroreductase is specifically expressed in the ventricular muscle under the control of vmhc promoter. Through treatment of the larvae at 3-4 dpf with Mtz [21], the authors killed the entire ventricular muscle cells and found that new ventricular cardiomyocytes arose from the area adjacent to the atrioventricular canal (AVC) and expanded across the chamber to restore the ventricular myocardium. Further lineage tracing indicated that $a m h c^{+}$atrial cardiomyocytes acquired ventricular muscle fate and migrated into the injured area to restore the lost ventricular muscle [48] (Figure 2B). This atrial-to-ventricular transdifferentiation was indirectly regulated by the endocardium through the Notch pathway [48]. However, this transdifferentiation phenomenon is only observed in the larvae when the heart is largely hurt, whether similar phenomenon could be recapitulated in adult fish requires further investigations.

Several molecules have been demonstrated to be essential for heart regeneration. Cxcl12a-Cxcr4b axis is critical in the cardiomyocyte migration during regeneration [49]. The dynamic deposition of extracellular matrix is required for the new myocardium restoration in the ventricular wall [50]. During the cardiomyocyte proliferation, clusters of molecular networks orchestrate together to ensure the integrity of regeneration. These factors play either positive or negative roles [14]. RA, Tgf, Pdgf , Hh, insulin-like growth factors and Jak-Stat pathway positively promote proliferation of cardiomyocytes [14]. In contrast, Notch pathway plays both promoting and inhibitory the roles, and balance between these activation and inhibition is important for the heart
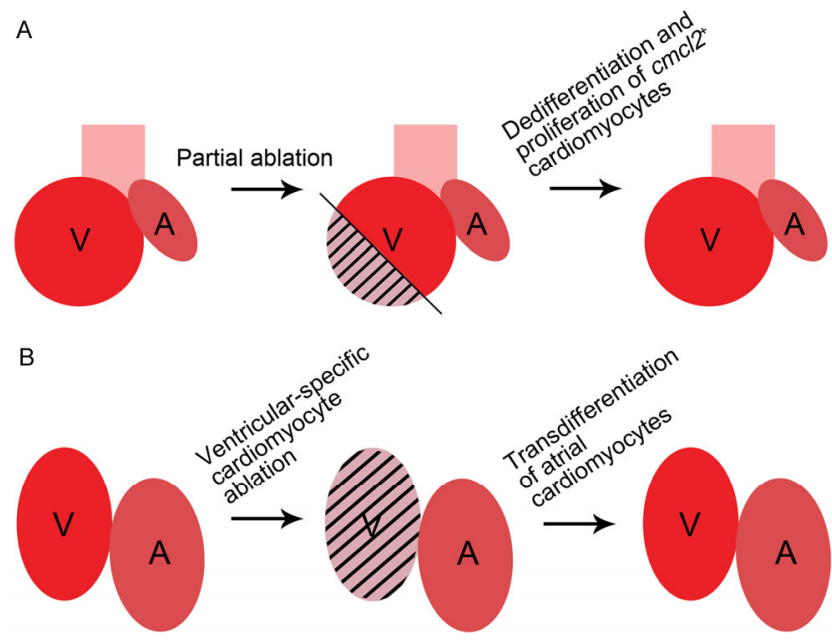

Figure 2 (color online) Two kinds of the regeneration models when the ventricle is differently ablated. A, When the adult ventricle is partially ablated, the resident $\mathrm{cmcl}^{+}$cardiomyocytes served as the regeneration source to dedifferentiate and proliferate to repair the injured ventricle. B, The atrial cardiomyocytes undergo transdifferentiation to restore the total ventricle after the ventricular-specific cardiomyocytes are killed in the embryonic zebrafish.

regeneration [51]. Meanwhile, the roles of environmental factors, in particular the oxygen level, have been investigated [52]. The result showed that low oxygen condition caused by heart failure after injury was an effective cue to promote cardiomyocyte proliferation. In contrast, hyperoxic conditions substantially inhibited the proliferation [52]. This regulation is mediated by hypoxia-inducible transcriptional factors (Hifs) [14]. Recently, $\mathrm{H}_{2} \mathrm{O}_{2}$ was found to act as a necessary and sufficient epicardial and myocardial signal to prime the heart regeneration, which is mediated by the destabilization of Dusp6 and increases in the phosphorylation of Erk signaling molecules [53]. The negative regulators include Mkk6-p38 MAPK pathway whose activation suppresses heart regeneration [14]. Recently, miR-133 that was involved in the fin regeneration was also indicated to be functional in the heart regeneration [54]. The study indicated that miR-133 repressed heart regeneration partially through the inhibition of Connexin 43 (Cx43) [54].

\subsection{CNS regeneration}

CNS is composed of various neurons and its regeneration capacity is poor in mammals. However, continual neurogenesis and regenerative capability have been demonstrated in zebrafish [13]. The studies of zebrafish CNS regeneration are mainly divided into three fields: adult neurons in the brain in particular in the telencephalon after physical lesion, spinal cord or axon restoration after truncation, and retina (photoreceptor) regeneration $[3,8,9,13]$. It is currently in general believed that the regenerative features of zebrafish CNS depend on the continually proliferative stem/progenitor cells and the permissive environments $[13,55,56]$. 
To understand the events and mechanisms of neuronal regeneration, the physical injury of the telencephalon is widely used in adult zebrafish. Through this approach and the Cre/loxP based lineage tracing, her4.1-positive ventricular radial glia progenitor cells were found to react to the injury, subsequently proliferate and generate neuroblasts that migrate to the lesion site [57]. Thus, the adult zebrafish brain has a remarkable potential of neuronal repair by endogenous adult stem/progenitor cells. However, how these stem/progenitor cells switched to the highly proliferative state required further investigations. Recent study indicated that these neuronal progenitor cells from different regions were endowed with different proliferative capability. The following migration of these progenitors to the injury site is regulated by the Notch signaling pathway [58]. However, the study of zebrafish brain regeneration is at the beginning stage, and key cellular as well as molecular mechanisms remain to be elucidated.

Another field lies in axon regeneration. In zebrafish, the regeneration assays of optic nerve and spinal cord after surgical interruption are well established and the results suggest the variable regrowth capacity in different types of axons [9,59-61]. Through the functional and behavior studies which reliably reflect the regeneration of axon after lesion, investigators found that the optic nerve re-established by 28-35 days post lesion $(\mathrm{dpl})$ and the related long-term synaptic rearrangements recovered after 3 months [59,62]. And the spinal cord regeneration took about 42 days [63-65]. Several extrinsic and intrinsic factors have been suggested to take part in the regeneration process, but further studies are still required [9]. In the spinal cord regeneration, ependymo-radial glial cells usually form bridges to re-connect the severed spinal cord $[9,63,66]$. During this process, the inhibitory components such as chondroitin sulfate proteoglycans (CSPGs) [67] and myelin-associated inhibitory molecules normally present in mammals, are absent in zebrafish [68]. Instead, the factors promoting axon growth such as contactins, P0, L1 related molecules, GAP-43, and alpha-1 tubulin are highly expressed in zebrafish after lesion [9,69-73]. Recently, ptena, but not ptenb was suggested to reduce the spinal cord regeneration [74], and Jak-Stat signaling was reported to induce optic nerve regeneration via overcoming the inhibitory socs 3 and $s f p q$ [75]. The proper reconnections of the axons to reach their targets over long distances are another amazing phenomenon of the axon regrowth. Although the degenerating tracts and other guidance molecules such as CSPGs, tenascin-R and ephirins perhaps play balanced roles in this process $[63,68,76]$, the mechanism is still under debate and requires further studies. Recently, the posterior lateral line nerve regeneration was used as an easy-followed platform to dissect the axon regeneration. Related studies suggested that the regenerating axons followed the path laid down by the original nerve branches, and regeneration-promoting factors induced by the first cut facilitated regeneration after a second cut $[9,77]$.

In addition, a number of studies have been performed in the regeneration of zebrafish retina, an important appendix of brain [8]. Up to now, a subpopulation of Müller glia has been identified as the origin of both rod progenitor lineage and the "neurogenic clusters" in regenerating fish retina [78-80]. Several molecules, in particular the Asc1a-Pax6 signaling axis, play important parts $[81,82]$. More information is currently not available and needs future studies.

\subsection{Liver regeneration}

Different to the organs mentioned above, the liver can regenerate both in mammals and in zebrafish. In mice, when $2 / 3$ of the liver are surgically removed (partial hepatectomy, PHx), it is able to completely regenerate [3]. However, more information is still required to understand the cellular and molecular mechanisms of liver regeneration. Knowledge of liver regeneration has been remarkably advanced using zebrafish, particularly in the screen of candidate chemicals that promotes liver regeneration. Several types of liver injury models have been established [3]. The PHx model in adult zebrafish is widely used and this model suggests that the regenerating liver originates from the healthy hepatocytes in the residual liver $[3,83,84]$ (Figure 3A). The cell cycle regulator uhrfl was indicated as an important regulator [85]. Additionally, the topoisomerase 2a, Wnt, Fgf and Bmp signaling pathways [3,84] play roles. Recently, through the chemical screen, PGE2 was identified as a hepatoprotective compound that limited liver damage $[84,86]$, and this function is mediated by the interaction between PGE2 and Wnt signaling [84,87]. Treatment of a novel GSNOR inhibitor (GSNORi) after PHx in adult fish enhanced cellular proliferation and regeneration [3,84,88]. The findings of PGE2 and NO signaling in the liver regeneration provide invaluable perspective for their clinic applications in the patients. Recently, Def-p53 pathway was proposed to suppress fibrotic scar formation to facilitate regeneration after PHx. This effect, at least in part, was mediated by the regulation of the pro-inflammatory factor, high-mobility group box $1[84,89]$. The antagonistic interplay between Wnt and Notch signaling crucially affected hepatocyte regeneration in the fibrotic liver using the ethanol-induced fibrotic zebrafish model [90]. To understand the liver regeneration when most, if not all, the hepatocytes are lost, several groups introduce the NTR-based genetic ablation of hepatocytes in the larvae $[21,91,92]$. These studies revealed origins of the regenerating hepatocytes and illustrated cell behaviors in the regeneration process (Figure 3B). These studies revealed that the biliary cells, including larger cholangiocytes, can transdifferentiate into hepatocytes and become the major contributors of regeneration after extreme hepatocyte loss (Figure 3B). This process required Notch signaling and the consequent activation of 


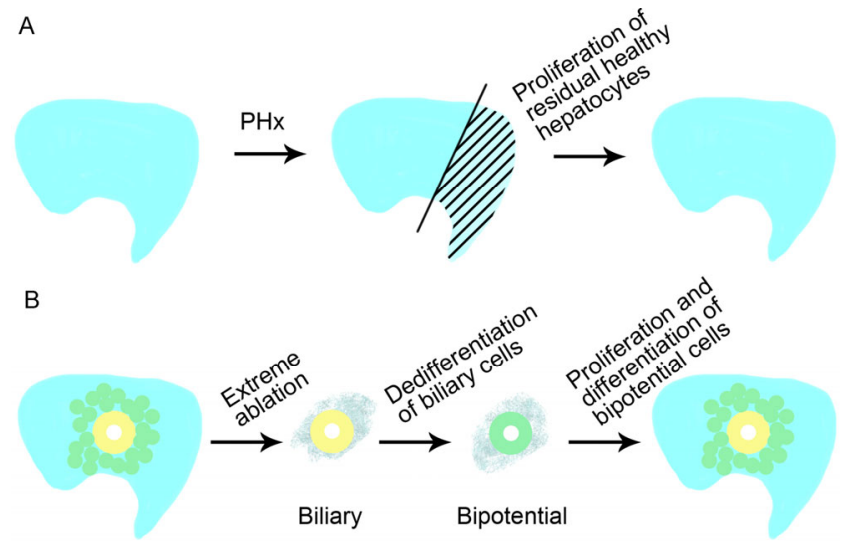

Figure 3 (color online) Two kinds of the regeneration progresses after the liver is differently ablated. A, When the liver is surgically removed (partial hepatectomy, $\mathrm{PHx}$ ), the residual healthy hepatocytes quickly proliferate to repair the injured liver. B, When the liver is extremely ablated, the biliary cells dedifferentiate to the bipotential cells, which in turn serve as the major contributors to regenerate the functional livers.

sox $9 b$ [91,92]. Whether other origins contribute to the regenerating liver needs further studies. All these findings provide the possibility to induce endogenous cell transdifferentiation as the major source of liver regeneration after extreme hepatocyte damage in mammals.

\section{Several questions to be answered}

\subsection{The reduced regenerative potential in higher ver- tebrates}

The reasons why the regenerative capacity, in particular that of brain, heart, limb, etc. reduces in the evolutionarily higher vertebrates, puzzled scientists for a long time. Although key factors are not well understood yet, several hypotheses have been proposed according to the related cues. One of such arguments is the difference of the stem/progenitor cells number and states in mammals versus zebrafish. It is also proposed that zebrafish possess active stem/progenitor cells in several organs and therefore their body size is able to continually increase with age $[93,94]$. In the brain, for example, the stem/progenitor cells are more widely distributed, which support neurons under physiological or regenerative circumstances $[13,95]$. Such capability may lose in mammals. The loss of continual growth ability in mammals may reflect the evolutionary advantage to reduce the danger of tumorigenesis. Therefore, tight control of cell proliferation is required but expenses the regenerative potential $[13,96]$. Another explanation is the absence of scar in zebrafish. Scar is a structure used by mammals to seal the injury site and prevent further damages. However, the scar also induces a block environment for the organ regeneration because of excess granulation tissue and disorganized collagen deposition in the scar tissues [13]. Other extrinsic environment factors including the extracellular matrix (ECM) may also be responsible. For example, in chronic liver failure patients with sustained fibrosis, excessive accumulation of ECM proteins substantially dampens the proliferative capacity of hepatocytes, resulting in poor prognosis and high mortality [90]. Similarly, lack of axon regrowth is brought about in part by a hostile growth environment containing growthinhibitory ECM components, such as chondroitin sulfate proteoglycans (CSPGs), and growth-inhibitory molecules produced by myelin or myelin debris [67]. The cell-intrinsic mechanisms regulating the cellular plasticity may have additional influences on the regeneration, and the impact may finally depend on the combination of cell-intrinsic and extrinsic factors [13]. The detailed mechanisms require further investigations in details.

\subsection{The different regulatory networks in development versus regeneration}

Whether organ regeneration is the recapitulation of organogenesis process is still controversial. Although several molecular networks, in particular Wnt, Notch, Hh, and Bmp, play indispensible roles in the cell lineage commitment, cell proliferation and differentiation in both development and regeneration [97], quite a number of studies showed their differences [98-100]. For example, the role of Gata3 following telencephalon stab wounding and caudal fin injury [101], and the specific expression of KLF6/7 in the axon regrowth, are absent in the corresponding organogenesis [102]. Therefore, whether there are special molecules essential for regeneration are quite important for our understanding of regeneration.

\subsection{Roles of inflammation and myeloid cells (macro- phage) in the regeneration}

Previous opinions mentioned that the injury-induced acute inflammation was the negative factor of regeneration $[103,104]$. However, this view was recently challenged by Nikos Kyritsis et al. [105], who demonstrated that acute inflammation was required and sufficient for increases in the proliferation of neural progenitors and subsequent neurogenesis in adult zebrafish brain after traumatic injury. And the inflammation induced cysteinyl leukotriene signaling was an essential component of this regeneration process [105]. This study prompts re-examinations of the roles of inflammation in the regeneration. It is not surprising that the leukocytes, in particular macrophages and neutrophils that characterize inflammation become modifiers of inflammation. Their roles in regeneration are another hot topic highly investigated in mammals. In truly regenerative rather than healing models, neutrophils have generally been considered as less critical contributors than macrophages or even viewed as impediments, although some views argued that neutrophils promoted axon regeneration in the optic nerve after a crush injury through high expression of oncomodulin $[106,107]$. Macrophages have been studied much more ex- 
tensively than neutrophils for their role as a regeneration modifier [106,108]. Many studies indicate that macrophages, in particular the M2 macrophage polarization, are involved in promoting regenerative over scarring outcomes after injury [109]. Recently, functions of macrophages and neutrophils in zebrafish organ regeneration, primarily in the fin regeneration, were analyzed [110,111]. These studies suggest that neutrophils are negative regulator of fin regeneration, whereas macrophages promote restoration of larval and adult fins after amputation $[110,111]$.

\section{Conclusion and perspectives}

Understanding of cellular and molecular mechanisms underlying organ regeneration is critical for the development of therapeutic method after organ damages. Considerable progression has already been made after the extensive investigations using several model organisms in recent years. However, an overall and deep understanding is still far away. The powerful regenerative capacity of zebrafish combined with several unique advantages substantially contributes to understanding of organ regeneration. Continuous efforts will further extend our knowledge, and will finally enable people to find out approaches to induce endogenous cells to restore the damaged organ in mammals, which is the destination we are looking forward to. Taking advantages of the technical advances, such as recently developed reverse genetic tools like TALEN and CRISPR/cas9 [112-114], we should positively expect that mechanisms underlying organ regeneration will eventually be revealed in the future.

1 Poss KD. Advances in understanding tissue regenerative capacity and mechanisms in animals. Nat Rev Genet, 2010, 11: 710-722

2 Tal TL, Franzosa JA, Tanguay RL. Molecular signaling networks that choreograph epimorphic fin regeneration in zebrafish - a mini-review. Gerontology, 2010, 56: 231-240

3 Goessling W, North TE. Repairing quite swimmingly: advances in regenerative medicine using zebrafish. Dis Model Mech, 2014, 7: 769-776

4 Grunwald DJ, Eisen JS. Headwaters of the zebrafish-emergence of a new model vertebrate. Nat Rev Genet, 2002, 3: 717-724

5 Driever W, Solnica-Krezel L, Schier AF, Neuhauss SC, Malicki J, Stemple DL, Stainier DY, Zwartkruis F, Abdelilah S, Rangini Z, Belak J, Boggs C. A genetic screen for mutations affecting embryogenesis in zebrafish. Development, 1996, 123: 37-46

6 Haffter P, Granato M, Brand M, Mullins MC, Hammerschmidt M, Kane DA, Odenthal J, van Eeden FJ, Jiang YJ, Heisenberg CP, Kelsh RN, Furutani-Seiki M, Vogelsang E, Beuchle D, Schach U, Fabian C, Nüsslein-Volhard C. The identification of genes with unique and essential functions in the development of the zebrafish, Danio rerio. Development, 1996, 123: 1-36

7 Stainier DY, Fouquet B, Chen JN, Warren KS, Weinstein BM, Meiler SE, Mohideen MA, Neuhauss SC, Solnica-Krezel L, Schier AF, Zwartkruis F, Stemple DL, Malicki J, Driever W, Fishman MC. Mutations affecting the formation and function of the cardiovascular system in the zebrafish embryo. Development, 1996, 123: 285-292

8 Brockerhoff SE, Fadool JM. Genetics of photoreceptor degeneration and regeneration in zebrafish. Cell Mol Life Sci, 2011, 68: 651-659
9 Becker T, Becker CG. Axonal regeneration in zebrafish. Curr Opin Neurobiol, 2014, 27C: 186-191

10 Poss KD, Wilson LG, Keating MT. Heart regeneration in zebrafish. Science, 2002, 298: 2188-2190

11 Poss KD, Keating MT, Nechiporuk A. Tales of regeneration in zebrafish. Dev Dyn, 2003, 226: 202-210

12 McCampbell KK, Wingert RA. New tides: using zebrafish to study renal regeneration. Transl Res, 2014, 163: 109-122,

13 Kizil C, Kaslin J, Kroehne V, Brand M. Adult neurogenesis and brain regeneration in zebrafish. Dev Neurobiol, 2012, 72: 429-461

14 Kikuchi K. Advances in understanding the mechanism of zebrafish heart regeneration. Stem Cell Res, 2014, 13: 542-555

15 Matrone G, Taylor JM, Wilson KS, Baily J, Love GD, Girkin JM, Mullins JJ, Tucker CS, Denvir MA. Laser-targeted ablation of the zebrafish embryonic ventricle: a novel model of cardiac injury and repair. Int J Cardiol, 2013, 168: 3913-3919

16 Kurita R, Sagara H, Aoki Y, Link BA, Arai K, Watanabe S. Suppression of lens growth by alphaA-crystallin promoter-driven expression of diphtheria toxin results in disruption of retinal cell organization in zebrafish. Dev Biol, 2003, 255: 113-127

17 Slanchev K, Stebler J, Cueva-Mendez G, Raz E. Development without germ cells: the role of the germ line in zebrafish sex differentiation. Proc Natl Acad Sci USA, 2005, 102: 4074-4079

18 Bulina ME, Chudakov DM, Britanova OV, Yanushevich YG, Staroverov DB, Chepurnykh TV, Merzlyak EM, Shkrob MA, Lukyanov S, Lukyanov KA. A genetically encoded photosensitizer. Nat Biotechnol, 2006, 24: 95-99

19 Pisharath H. Validation of nitroreductase, a prodrug-activating enzyme, mediated cell death in embryonic zebrafish (Danio rerio). Comp Med, 2007, 57: 241-246

20 Curado S, Stainier DY, Anderson RM. Nitroreductase-mediated cell/tissue ablation in zebrafish: a spatially and temporally controlled ablation method with applications in developmental and regeneration studies. Nat Protoc, 2008, 3: 948-954

21 Curado S, Anderson RM, Jungblut B, Mumm J, Schroeter E, Stainier DY. Conditional targeted cell ablation in zebrafish: a new tool for regeneration studies. Dev Dyn, 2007, 236: 1025-1035

22 Lush ME, Piotrowski T. Sensory hair cell regeneration in the zebrafish lateral line. Dev Dyn, 2014, 243: 1187-1202

23 Nechiporuk A, Keating MT. A proliferation gradient between proximal and msxb-expressing distal blastema directs zebrafish fin regeneration. Development, 2002, 129: 2607-2617

24 Akimenko MA, Mari-Beffa M, Becerra J, Geraudie J. Old questions, new tools, and some answers to the mystery of fin regeneration. Dev Dyn, 2003, 226: 190-201

25 Iovine MK. Conserved mechanisms regulate outgrowth in zebrafish fins. Nat Chem Biol, 2007, 3: 613-618

26 Singh SP, Holdway JE, Poss KD. Regeneration of amputated zebrafish fin rays from de novo osteoblasts. Dev Cell, 2012, 22: 879-886

27 Geurtzen K, Knopf F, Wehner D, Huitema LF, Schulte-Merker S, Weidinger G. Mature osteoblasts dedifferentiate in response to traumatic bone injury in the zebrafish fin and skull. Development, 2014, 141: 2225-2234

28 Kawakami Y, Rodriguez EC, Raya M, Kawakami H, Marti M, Dubova I, Izpisua Belmonte JC. Wnt/beta-catenin signaling regulates vertebrate limb regeneration. Genes Dev, 2006, 20: 3232-3237

29 Poss KD, Shen J, Keating MT. Induction of lefl during zebrafish fin regeneration. Dev Dyn, 2000, 219: 282-286

30 Stoick-Cooper CL, Weidinger G, Riehle KJ, Hubbert C, Major MB, Fausto N, Moon RT. Distinct Wnt signaling pathways have opposing roles in appendage regeneration. Development, 2007, 134: 479-489

31 Mathew LK, Sengupta S, Kawakami A, Andreasen EA, Lohr CV, Loynes CA, Renshaw SA, Peterson RT, Tanguay RL. Unraveling tissue regeneration pathways using chemical genetics. J Biol Chem, 2007, 282: 35202-35210

32 Chen B, Dodge ME, Tang W, Lu J, Ma Z, Fan CW, Wei S, Hao W, Kilgore J, Williams NS, Roth MG, Amatruda JF, Chen C, Lum L. Small molecule-mediated disruption of Wnt-dependent signaling in tissue regeneration and cancer. Nat Chem Biol, 2009, 5: 100-107 
33 Lee Y, Grill S, Sanchez A, Murphy-Ryan M, Poss KD. Fgf signaling instructs position-dependent growth rate during zebrafish fin regeneration. Development, 2005, 132: 5173-5183

34 Whitehead GG, Makino S, Lien CL, Keating MT. fgf20 is essential for initiating zebrafish fin regeneration. Science, 2005, 310: 1957-1960

35 Geraudie J, Monnot MJ, Brulfert A, Ferretti P. Caudal fin regeneration in wild type and long-fin mutant zebrafish is affected by retinoic acid. Int J Dev Biol, 1995, 39: 373-381

36 White JA, Boffa MB, Jones B, Petkovich M. A zebrafish retinoic acid receptor expressed in the regenerating caudal fin. Development, 1994, 120: 1861-1872

37 Wehner D, Cizelsky W, Vasudevaro MD, Ozhan G, Haase C, Kagermeier-Schenk B, Roder A, Dorsky RI, Moro E, Argenton F, Kühl M, Weidinger G. Wnt/beta-catenin signaling defines organizing centers that orchestrate growth and differentiation of the regenerating zebrafish caudal fin. Cell Rep, 2014, 6: 467-481

38 Thatcher EJ, Paydar I, Anderson KK, Patton JG. Regulation of zebrafish fin regeneration by microRNAs. Proc Natl Acad Sci USA, 2008, 105: 18384-18389

39 Yin VP, Thomson JM, Thummel R, Hyde DR, Hammond SM, Poss KD. Fgf-dependent depletion of microRNA-133 promotes appendage regeneration in zebrafish. Genes Dev, 2008, 22: 728-733

40 Yin VP, Poss KD. New regulators of vertebrate appendage regeneration. Curr Opin Genet Dev, 2008, 18: 381-386

41 Varga M, Sass M, Papp D, Takacs-Vellai K, Kobolak J, Dinnyes A, Klionsky DJ, Vellai T. Autophagy is required for zebrafish caudal fin regeneration. Cell Death Differ, 2014, 21: 547-556

42 Kujawski S, Lin W, Kitte F, Bormel M, Fuchs S, Arulmozhivarman G, Vogt S, Theil D, Zhang Y, Antos CL. Calcineurin regulates coordinated outgrowth of zebrafish regenerating fins. Dev Cell, 2014, 28: 573-587

43 Gupta V, Poss KD. Clonally dominant cardiomyocytes direct heart morphogenesis. Nature, 2012, 484: 479-484

44 Jopling C, Sleep E, Raya M, Marti M, Raya A, Izpisua Belmonte JC. Zebrafish heart regeneration occurs by cardiomyocyte dedifferentiation and proliferation. Nature, 2010, 464: 606-609

45 Kikuchi K, Holdway JE, Werdich AA, Anderson RM, Fang Y, Egnaczyk GF, Evans T, Macrae CA, Stainier DY, Poss KD. Primary contribution to zebrafish heart regeneration by gata $^{+}$cardiomyocytes. Nature, 2010, 464: 601-605

46 Gonzalez-Rosa JM, Peralta M, Mercader N. Pan-epicardial lineage tracing reveals that epicardium derived cells give rise to myofibroblasts and perivascular cells during zebrafish heart regeneration. Dev Biol, 2012, 370: 173-186

47 Kikuchi K, Gupta V, Wang J, Holdway JE, Wills AA, Fang Y, Poss KD. $t c f 21^{+}$epicardial cells adopt non-myocardial fates during zebrafish heart development and regeneration. Development, 2011, 138: 2895-2902

48 Zhang R, Han P, Yang H, Ouyang K, Lee D, Lin YF, Ocorr K, Kang $\mathrm{G}$, Chen J, Stainier DY, Yelon D, Chi NC. In vivo cardiac reprogramming contributes to zebrafish heart regeneration. Nature, 2013, 498: 497-501

49 Itou J, Oishi I, Kawakami H, Glass TJ, Richter J, Johnson A, Lund TC, Kawakami Y. Migration of cardiomyocytes is essential for heart regeneration in zebrafish. Development, 2012, 139: 4133-4142

50 Wang J, Karra R, Dickson AL, Poss KD. Fibronectin is deposited by injury-activated epicardial cells and is necessary for zebrafish heart regeneration. Dev Biol, 2013, 382: 427-435

51 Zhao L, Borikova AL, Ben-Yair R, Guner-Ataman B, MacRae CA, Lee RT, Burns CG, Burns CE. Notch signaling regulates cardiomyocyte proliferation during zebrafish heart regeneration. Proc Natl Acad Sci USA, 2014, 111: 1403-1408

52 Jopling C, Sune G, Faucherre A, Fabregat C, Izpisua Belmonte JC. Hypoxia induces myocardial regeneration in zebrafish. Circulation, 2012, 126: 3017-3027

53 Han P, Zhou XH, Chang N, Xiao CL, Yan S, Ren H, Yang XZ, Zhang ML, Wu Q, Tang B, Diao JP, Zhu X, Zhang C, Li CY, Cheng $\mathrm{H}$, Xiong JW. Hydrogen peroxide primes heart regeneration with a derepression mechanism. Cell Res, 2014, 24: 1091-1107
54 Yin VP, Lepilina A, Smith A, Poss KD. Regulation of zebrafish heart regeneration by miR-133. Dev Biol, 2012, 365: 319-327

55 Becker CG, Becker T. Adult zebrafish as a model for successful central nervous system regeneration. Restor Neurol Neurosci, 2008, 26: $71-80$

56 Fleisch VC, Fraser B, Allison WT. Investigating regeneration and functional integration of CNS neurons: lessons from zebrafish genetics and other fish species. Biochim Biophys Acta, 2011, 1812: 364-380

57 Kroehne V, Freudenreich D, Hans S, Kaslin J, Brand M. Regeneration of the adult zebrafish brain from neurogenic radial gliatype progenitors. Development, 2011, 138: 4831-4841

58 Kishimoto N, Shimizu K, Sawamoto K. Neuronal regeneration in a zebrafish model of adult brain injury. Dis Model Mech, 2012, 5: 200-209

59 Zou S, Tian C, Ge S, Hu B. Neurogenesis of retinal ganglion cells is not essential to visual functional recovery after optic nerve injury in adult zebrafish. PLoS One, 2013, 8: e57280

60 Fujikawa C, Nagashima M, Mawatari K, Kato S. HSP70 gene expression in the zebrafish retina after optic nerve injury: a comparative study under heat shock stresses. Adv Exp Med Biol, 2012, 723: 663-668

61 Nagashima M, Fujikawa C, Mawatari K, Mori Y, Kato S. HSP70, the earliest-induced gene in the zebrafish retina during optic nerve regeneration: its role in cell survival. Neurochem Int, 2011, 58: 888-895

62 Kato S, Matsukawa T, Koriyama Y, Sugitani K, Ogai K. A molecular mechanism of optic nerve regeneration in fish: the retinoid signaling pathway. Prog Retin Eye Res, 2013, 37: 13-30

63 Goldshmit Y, Sztal TE, Jusuf PR, Hall TE, Nguyen-Chi M, Currie PD. Fgf-dependent glial cell bridges facilitate spinal cord regeneration in zebrafish. J Neurosci, 2012, 32: 7477-7492

64 Becker T, Wullimann MF, Becker CG, Bernhardt RR, Schachner M. Axonal regrowth after spinal cord transection in adult zebrafish. $\mathrm{J}$ Comp Neurol, 1997, 377: 577-595

65 Dias TB, Yang YJ, Ogai K, Becker T, Becker CG. Notch signaling controls generation of motor neurons in the lesioned spinal cord of adult zebrafish. J Neurosci, 2012, 32: 3245-3252

66 Kuscha V, Frazer SL, Dias TB, Hibi M, Becker T, Becker CG. Lesion-induced generation of interneuron cell types in specific dorsoventral domains in the spinal cord of adult zebrafish. J Comp Neurol, 2012, 520: 3604-3616

67 Fawcett JW, Schwab ME, Montani L, Brazda N, Muller HW. Defeating inhibition of regeneration by scar and myelin components. Handb Clin Neurol, 2012, 109: 503-522

68 Becker CG, Becker T. Repellent guidance of regenerating optic axons by chondroitin sulfate glycosaminoglycans in zebrafish. J Neurosci, 2002, 22: 842-853

69 Schweitzer J, Gimnopoulos D, Lieberoth BC, Pogoda HM, Feldner J, Ebert A, Schachner M, Becker T, Becker CG. Contactin1a expression is associated with oligodendrocyte differentiation and axonal regeneration in the central nervous system of zebrafish. Mol Cell Neurosci, 2007, 35: 194-207

70 Schweitzer J, Becker T, Becker CG, Schachner M. Expression of protein zero is increased in lesioned axon pathways in the central nervous system of adult zebrafish. Glia, 2003, 41: 301-317

71 Kusik BW, Hammond DR, Udvadia AJ. Transcriptional regulatory regions of gap43 needed in developing and regenerating retinal ganglion cells. Dev Dyn, 2010, 239: 482-495

72 Becker CG, Lieberoth BC, Morellini F, Feldner J, Becker T, Schachner M. L1.1 is involved in spinal cord regeneration in adult zebrafish. J Neurosci, 2004, 24: 7837-7842

73 Veldman MB, Bemben MA, Thompson RC, Goldman D. Gene expression analysis of zebrafish retinal ganglion cells during optic nerve regeneration identifies KLF6a and KLF7a as important regulators of axon regeneration. Dev Biol, 2007, 312: 596-612

74 Liu D, Yu Y, Schachner M. Ptena, but not Ptenb, reduces regeneration after spinal cord injury in adult zebrafish. Exp Neurol, 2014, 261C: 196-205

75 Elsaeidi F, Bemben MA, Zhao XF, Goldman D. Jak/Stat signaling 
stimulates zebrafish optic nerve regeneration and overcomes the inhibitory actions of Socs3 and Sfpq. J Neurosci, 2014, 34: 2632-2644

76 Becker CG, Schweitzer J, Feldner J, Schachner M, Becker T. Tenascin-R as a repellent guidance molecule for newly growing and regenerating optic axons in adult zebrafish. Mol Cell Neurosci, 2004, 26: 376-389

77 Graciarena M, Dambly-Chaudiere C, Ghysen A. Dynamics of axonal regeneration in adult and aging zebrafish reveal the promoting effect of a first lesion. Proc Natl Acad Sci USA, 2014, 111: 1610-1615

78 Bernardos RL, Barthel LK, Meyers JR, Raymond PA. Late-stage neuronal progenitors in the retina are radial Muller glia that function as retinal stem cells. J Neurosci, 2007, 27: 7028-7040

79 Fimbel SM, Montgomery JE, Burket CT, Hyde DR. Regeneration of inner retinal neurons after intravitreal injection of ouabain in zebrafish. J Neurosci, 2007, 27: 1712-1724

80 Thummel R, Kassen SC, Enright JM, Nelson CM, Montgomery JE, Hyde DR. Characterization of Muller glia and neuronal progenitors during adult zebrafish retinal regeneration. Exp Eye Res, 2008, 87: 433-444

81 Fausett BV, Goldman D. A role for alpha1 tubulin-expressing Muller glia in regeneration of the injured zebrafish retina. J Neurosci, 2006, 26: 6303-6313

82 Thummel R, Enright JM, Kassen SC, Montgomery JE, Bailey TJ, Hyde DR. Pax6a and Pax6b are required at different points in neuronal progenitor cell proliferation during zebrafish photoreceptor regeneration. Exp Eye Res, 2010, 90: 572-582

83 Curado S, Stainier DY. deLiver'in regeneration: injury response and development. Semin Liver Dis, 2010, 30: 288-295

84 Kan NG, Junghans D, Izpisua Belmonte JC. Compensatory growth mechanisms regulated by BMP and FGF signaling mediate liver regeneration in zebrafish after partial hepatectomy. FASEB J, 2009, 23: 3516-3525

85 Sadler KC, Krahn KN, Gaur NA, Ukomadu C. Liver growth in the embryo and during liver regeneration in zebrafish requires the cell cycle regulator, uhrf1. Proc Natl Acad Sci USA, 2007, 104: $1570-1575$

86 North TE, Babu IR, Vedder LM, Lord AM, Wishnok JS, Tannenbaum SR, Zon LI, Goessling W. PGE2-regulated wnt signaling and $\mathrm{N}$-acetylcysteine are synergistically hepatoprotective in zebrafish acetaminophen injury. Proc Natl Acad Sci USA, 2010, 107: 17315-17320

87 Goessling W, North TE, Loewer S, Lord AM, Lee S, Stoick-Cooper CL, Weidinger G, Puder M, Daley GQ, Moon RT, Zon LI. Genetic interaction of PGE2 and Wnt signaling regulates developmental specification of stem cells and regeneration. Cell, 2009, 136: 1136-1147

88 Cox AG, Saunders DC, Kelsey PB Jr., Conway AA, Tesmenitsky Y, Marchini JF, Brown KK, Stamler JS, Colagiovanni DB, Rosenthal GJ, Croce KJ, North TE, Goessling W. S-nitrosothiol signaling regulates liver development and improves outcome following toxic liver injury. Cell Rep, 2014, 6: 56-69

89 Zhu Z, Chen J, Xiong JW, Peng J. Haploinsufficiency of Def activates p53-dependent TGFbeta signalling and causes scar formation after partial hepatectomy. PLoS One, 2014, 9: e96576

90 Huang M, Chang A, Choi M, Zhou D, Anania FA, Shin CH. Antagonistic interaction between Wnt and Notch activity modulates the regenerative capacity of a zebrafish fibrotic liver model. Hepatology, 2014, 60: 1753-1766

91 Choi TY, Ninov N, Stainier DY, Shin D. Extensive conversion of hepatic biliary epithelial cells to hepatocytes after near total loss of hepatocytes in zebrafish. Gastroenterology, 2014, 146: 776-788

$92 \mathrm{He} \mathrm{J,} \mathrm{Lu} \mathrm{H,} \mathrm{Zou} \mathrm{Q,} \mathrm{Luo} \mathrm{L.} \mathrm{Regeneration} \mathrm{of} \mathrm{liver} \mathrm{after} \mathrm{extreme}$ hepatocyte loss occurs mainly via biliary transdifferentiation in zebrafish. Gastroenterology, 2014, 146: 789-800

93 Goldsmith MI, Iovine MK, O'Reilly-Pol T, Johnson SL. A developmental transition in growth control during zebrafish caudal fin development. Dev Biol, 2006, 296: 450-457

94 Tsai SB, Tucci V, Uchiyama J, Fabian NJ, Lin MC, Bayliss PE, Neuberg DS, Zhdanova IV, Kishi S. Differential effects of genotoxic stress on both concurrent body growth and gradual senescence in the adult zebrafish. Aging Cell, 2007, 6: 209-224

95 Grandel H, Kaslin J, Ganz J, Wenzel I, Brand M. Neural stem cells and neurogenesis in the adult zebrafish brain: origin, proliferation dynamics, migration and cell fate. Dev Biol, 2006, 295: 263-277

96 Pearson BJ, Sanchez AA. Regeneration, stem cells, and the evolution of tumor suppression. Cold Spring Harb Symp Quant Biol, 2008, 73: 565-572

97 Sanchez AA, Yamanaka S. Rethinking differentiation: stem cells, regeneration, and plasticity. Cell, 2014, 157: 110-119

98 Kizil C, Otto GW, Geisler R, Nusslein-Volhard C, Antos CL. Simplet controls cell proliferation and gene transcription during zebrafish caudal fin regeneration. Dev Biol, 2009, 325: 329-340

99 Millimaki BB, Sweet EM, Riley BB. Sox2 is required for maintenance and regeneration, but not initial development, of hair cells in the zebrafish inner ear. Dev Biol, 2010, 338: 262-269

100 Stewart S, Tsun ZY, Izpisua Belmonte JC. A histone demethylase is necessary for regeneration in zebrafish. Proc Natl Acad Sci USA, 2009, 106: 19889-19894

101 Kizil C, Kyritsis N, Dudczig S, Kroehne V, Freudenreich D, Kaslin J, Brand M. Regenerative neurogenesis from neural progenitor cells requires injury-induced expression of Gata3. Dev Cell, 2012, 23: 1230-1237

102 Veldman MB, Bemben MA, Thompson RC, Goldman D. Gene expression analysis of zebrafish retinal ganglion cells during optic nerve regeneration identifies KLF6a and KLF7a as important regulators of axon regeneration. Dev Biol, 2007, 312: 596-612

103 Hoehn BD, Palmer TD, Steinberg GK. Neurogenesis in rats after focal cerebral ischemia is enhanced by indomethacin. Stroke, 2005, 36: $2718-2724$

104 Iosif RE, Ekdahl CT, Ahlenius H, Pronk CJ, Bonde S, Kokaia Z, Jacobsen SE, Lindvall O. Tumor necrosis factor receptor 1 is a negative regulator of progenitor proliferation in adult hippocampal neurogenesis. J Neurosci, 2006, 26: 9703-9712

105 Kyritsis N, Kizil C, Zocher S, Kroehne V, Kaslin J, Freudenreich D, Iltzsche A, Brand M. Acute inflammation initiates the regenerative response in the adult zebrafish brain. Science, 2012, 338: 1353-1356

106 Keightley MC, Wang CH, Pazhakh V, Lieschke GJ. Delineating the roles of neutrophils and macrophages in zebrafish regeneration models. Int J Biochem Cell Biol, 2014, 56C: 92-106

107 Kurimoto T, Yin Y, Habboub G, Gilbert HY, Li Y, Nakao S, Hafezi-Moghadam A, Benowitz LI. Neutrophils express oncomodulin and promote optic nerve regeneration. J Neurosci, 2013, 33: 14816-14824

108 Chazaud B. Macrophages: supportive cells for tissue repair and regeneration. Immunobiology, 2014, 219: 172-178

109 Mosser DM, Edwards JP. Exploring the full spectrum of macrophage activation. Nat Rev Immunol, 2008, 8: 958-969

110 Li L, Yan B, Shi YQ, Zhang WQ, Wen ZL. Live imaging reveals differing roles of macrophages and neutrophils during zebrafish tail fin regeneration. J Biol Chem, 2012, 287: 25353-25360

111 Petrie TA, Strand NS, Tsung-Yang C, Rabinowitz JS, Moon RT. Macrophages modulate adult zebrafish tail fin regeneration. Development, 2014, 141: 2581-2591

112 Huang $\mathrm{P}$, Xiao A, Tong X, Zu Y, Wang Z, Zhang B. TALEN construction via "Unit Assembly" method and targeted genome modifications in zebrafish. Methods, 2014, 69: 67-75

113 Chang N, Sun C, Gao L, Zhu D, Xu X, Zhu X, Xiong JW, Xi JJ. Genome editing with RNA-guided Cas9 nuclease in zebrafish embryos. Cell Res, 2013, 23: 465-472

114 Zhang L, Zhou Q. CRISPR/Cas technology: a revolutionary approach for genome engineering. Sci China Life Sci, 2014, 57: 639-640

Open Access This article is distributed under the terms of the Creative Commons Attribution License which permits any use, distribution, and reproduction in any medium, provided the original author(s) and source are credited. 